\title{
The Influence of Impurity Content on Thermal Stability of Low Stacking Fault Energy Silver Processed by Severe Plastic Deformation
}

\author{
Z. Hegedüs ${ }^{1, a}$, J. Gubicza ${ }^{1, b}$, M. Kawasaki ${ }^{2, c}$, N.Q. Chinh ${ }^{1, d}$, Zs. Fogarassy ${ }^{3, e}$, \\ T.G. Langdon $2,4, f$
}

${ }^{1}$ Department of Materials Physics, Eötvös Loránd University, Pázmány Péter sétány 1/A, H-1117 Budapest, Hungary

${ }^{2}$ Department of Aerospace \& Mechanical Engineering and Materials Science, University of Southern California, Los Angeles, CA 90089-1453, USA

${ }^{3}$ Research Institute for Technical Physics and Materials Science, P.O.Box 49, H-1525 Budapest, Hungary

${ }^{4}$ Materials Research Group, School of Engineering Sciences, University of Southampton, Southampton SO17 1BJ, UK

azoltan885@yahoo.com (corresponding author), bgubicza@metal.elte.hu, ${ }^{\mathrm{b}} \mathrm{mkawasak@usc.edu,}$

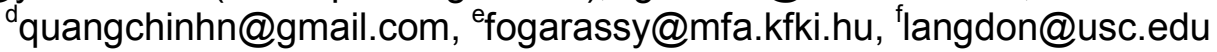

Keywords: Silver, Stacking fault energy, Equal-Channel Angular Pressing, Dislocations, Twins, Thermal stability.

\begin{abstract}
The effect of the impurity content on the evolution of the ultrafine-grained (UFG) microstructure in low stacking fault energy Ag and its stability at room and elevated temperatures were investigated. Samples of silver having high (99.995\%) and somewhat lower (99.99\%) purity levels were processed by equal-channel angular pressing (ECAP) at room temperature (RT) up to 16 passes. Although, the minimum grain size achieved by ECAP was $\sim 200 \mathrm{~nm}$ for both series, the lattice defect structure was strongly influenced by the impurity content. In the samples processed by 4-16 passes of ECAP a self-annealing occurred during storage RT that was promoted by the higher twin boundary frequency. Both room- and high-temperature thermal stability of $99.99 \%$ purity Ag were much better due to the pinning effect of impurities. It was found that a large number of dislocation loops remained in the microstructure even after recrystallization at high temperatures.
\end{abstract}

\section{Introduction}

Severe plastic deformation (SPD) methods are very effective procedures for processing bulk ultrafine-grained (UFG) metals [1,2]. One of the most frequently used methods is equal-channel angular pressing (ECAP) [1] where it is possible to produce bulk UFG materials having dimensions of several centimeters in all directions. The high strength of the ECAP-processed metals is attributed to the small grain size and the high dislocation density [3]. The thermal stability of the UFG microstructures is a very important issue from the point of view of their practical applications as if a recovery or recrystallization occurred, the high strength would be reduced. It has been shown recently that pure Ag samples processed by ECAP were very unstable due to their low stacking fault energy (SFE) and the UFG microstructure was self-annealed even at room temperature (RT) [4]. It is well known that impurities can hinder recovery and recrystallization due to their pinning effect on lattice defects, such as dislocations or grain-boundaries [5,6]. In this paper, the effect of impurity content on the stability of the UFG microstructure in Ag at RT and high temperature is studied. The latter experiments were carried out in a differential scanning calorimeter (DSC).

\section{Materials and Experimental Procedures}

Samples of silver of 99.99 at.\% (4N) and 99.995 at.\% (4N5) purity were manufactured by American Elements and ESPI Metals, respectively. The alloying elements and their concentrations are listed in Table 1. For both compositions, billets having lengths of approx. $70 \mathrm{~mm}$ and diameters of $10 \mathrm{~mm}$ 
were annealed for 1 hour at $741 \mathrm{~K}$ and then they were processed through 1, 4, 8 and 16 passes of ECAP at RT with a pressing velocity of $8 \mathrm{~mm} \cdot \mathrm{s}^{-1}$. The ECAP was performed using route $\mathrm{B}_{\mathrm{c}}$ where the billet is rotated in the same sense by $90^{\circ}$ around its longitudinal axis after each pass [7]. The ECAP die had an internal channel angle of $90^{\circ}$ and an outer arc of curvature of $20^{\circ}$. In this configuration one pass corresponds to an equivalent strain of $\sim 1[8]$.

The microstructures of the ECAP-processed specimens were investigated by X-ray line profile analysis on transverse sections cut perpendicular to the axes of the billets. The measurements of the $\mathrm{X}$-ray diffraction lines were performed using a special high-resolution diffractometer (Nonius FR591) with $\mathrm{CuK} \alpha_{1}$ radiation having a wavelength of $\lambda=0.15406 \mathrm{~nm}$. The line profiles were evaluated using the extended Convolutional Multiple Whole Profile (eCMWP) fitting procedure $[9,10]$. In this method, the diffraction pattern is fitted by the sum of a background spline and the convolution of the theoretical line profiles related to the crystallite size, dislocations and twin faults. This procedure gives the dislocation density and the twin-boundary frequency with good statistics. The twin-boundary frequency is defined as the fraction of twin boundaries among the $\{111\}$ lattice planes. The grain structure was examined using a FEI Quanta 3D scanning electron microscope (SEM) and a Philips CM-20 transmission electron microscope (TEM) operating at $200 \mathrm{kV}$. The TEM samples were mechanically thinned to $\sim 80 \mu \mathrm{m}$, cooled to liquid nitrogen temperature and then thinned with $6 \mathrm{keV} \mathrm{Ar}^{+}$ions from both sides until perforation. Finally, a thin damaged layer was removed using $2 \mathrm{keV} \mathrm{Ar}^{+}$ions.

Table 1. The alloying element contents for 4N and 4N5 purity samples. The concentration values supplied by the manufacturers are given in ppm.

\begin{tabular}{|c|c|c|c|c|c|c|c|c|c|}
\hline Purity & $\mathrm{Cu}$ & $\mathrm{Pb}$ & $\mathrm{Fe}$ & $\mathrm{Se}$ & $\mathrm{Ir}$ & $\mathrm{Au}$ & $\mathrm{Pd}$ & $\mathrm{Sb}$ & $\mathrm{Bi}$ \\
\hline 4N5 & 13 & 14 & 5 & 6 & 6 & 10 & 2 & - & - \\
\hline $4 \mathrm{~N}$ & 30 & 10 & 10 & 10 & - & - & - & 10 & 20 \\
\hline
\end{tabular}

Hardness measurements were carried out on samples cut perpendicular to the longitudinal axis of the ECAP-processed billets. The hardness was measured using a Zwick Roell $\mathrm{ZH} \mu$ Vickers microhardness indenter with a maximum applied load of $2000 \mathrm{mN}$ and a dwell time of $10 \mathrm{~s}$. The hardness of each sample was obtained as an average of at least 8 values determined along the diameter of the specimen. It is noted that systematic radius-dependence of the hardness was not observed.

The samples were stored at RT and hardness testing as well as X-ray line profile analysis were performed monthly in order to monitor the self-annealing of the samples. The thermal stability at elevated temperatures was investigated by DSC using a Perkin Elmer (DSC2) calorimeter with a heating rate of $10 \mathrm{~K} / \mathrm{min}$ up to $600 \mathrm{~K}$. The heat released during annealing was determined as the area under the exothermic peak detected in DSC experiments.

\section{Results and Discussion}

Microstructure After Processing by ECAP. The initial grain size was $\sim 60 \mu \mathrm{m}$ for both $4 \mathrm{~N} 5$ and $4 \mathrm{~N}$ purity Ag samples before ECAP-processing as determined by SEM. After one pass of ECAP it was reduced to $\sim 20 \mu \mathrm{m}$. After four passes the mean grain size was about $200 \mathrm{~nm}$ for both impurity levels and did not change considerably in further ECAP-processing. As an example Figs. 1a and $b$ show TEM images taken on 4N5 and 4N purity samples, respectively, processed by 8 ECAP passes. It can be concluded that the minimum grain size in Ag achievable by ECAP at RT is not very sensitive to the impurity level in the range between $4 \mathrm{~N} 5$ and $4 \mathrm{~N}$.

The dislocation density and the twin boundary frequency were determined by X-ray line profile analysis and plotted in Figs. 2a and $\mathrm{b}$, respectively. After one pass the dislocation density was higher for $4 \mathrm{~N}$ purity $\mathrm{Ag}\left(2.6 \pm 0.4 \times 10^{15} \mathrm{~m}^{-2}\right)$ than for the $4 \mathrm{~N} 5$ purity counterpart $\left(1.6 \pm 0.2 \times 10^{15}\right.$ $\mathrm{m}^{-2}$ ). However, the evolution of the dislocation density during further passes was similar for both 
impurity concentrations (see Fig. 2a). It increased up to 8 passes, reaching a very large saturation value of $4.6 \pm 0.5 \times 10^{15} \mathrm{~m}^{-2}$, then it decreased between 8 and 16 passes. The saturation value is exceptionally high compared to other pure face centered cubic (fcc) metals [11], which can be explained by the hindered annihilation of the highly dissociated dislocations in Ag having low SFE. It is well known that lattice dislocations in fcc structures dissociate into partials, having an equilibrium splitting distance inversely proportional to SFE. Since SFE is very low in Ag (16-22 $\left.\mathrm{mJ} \cdot \mathrm{m}^{-2}[12,13]\right)$, it leads to a very high splitting distance between partial dislocations.

Contrary to dislocation density, the evolution of twin boundary frequency is different for the two impurity levels (see Fig. 2b). In 4N5 purity Ag the twin boundary frequency increased monotonously up to 16 passes while for $4 \mathrm{~N}$ purity specimens it decreased after 4 passes. This difference can be explained by the various impurity levels of the two materials. After 4 passes the grain size was reduced to $\sim 200 \mathrm{~nm}$, and in this range twins are mainly nucleated at the grain boundaries [14]. Since impurities are usually segregated at the grain boundaries in UFG microstructures [15], therefore the higher impurity content in $4 \mathrm{~N} \mathrm{Ag}$ more effectively hindered the nucleation of twins and due to the interaction between dislocations and twins untwining occurred inside the grains during ECAP. This effect led to a decrease in the twin boundary frequency for more than 4 passes in the case of $4 \mathrm{~N}$ purity Ag.
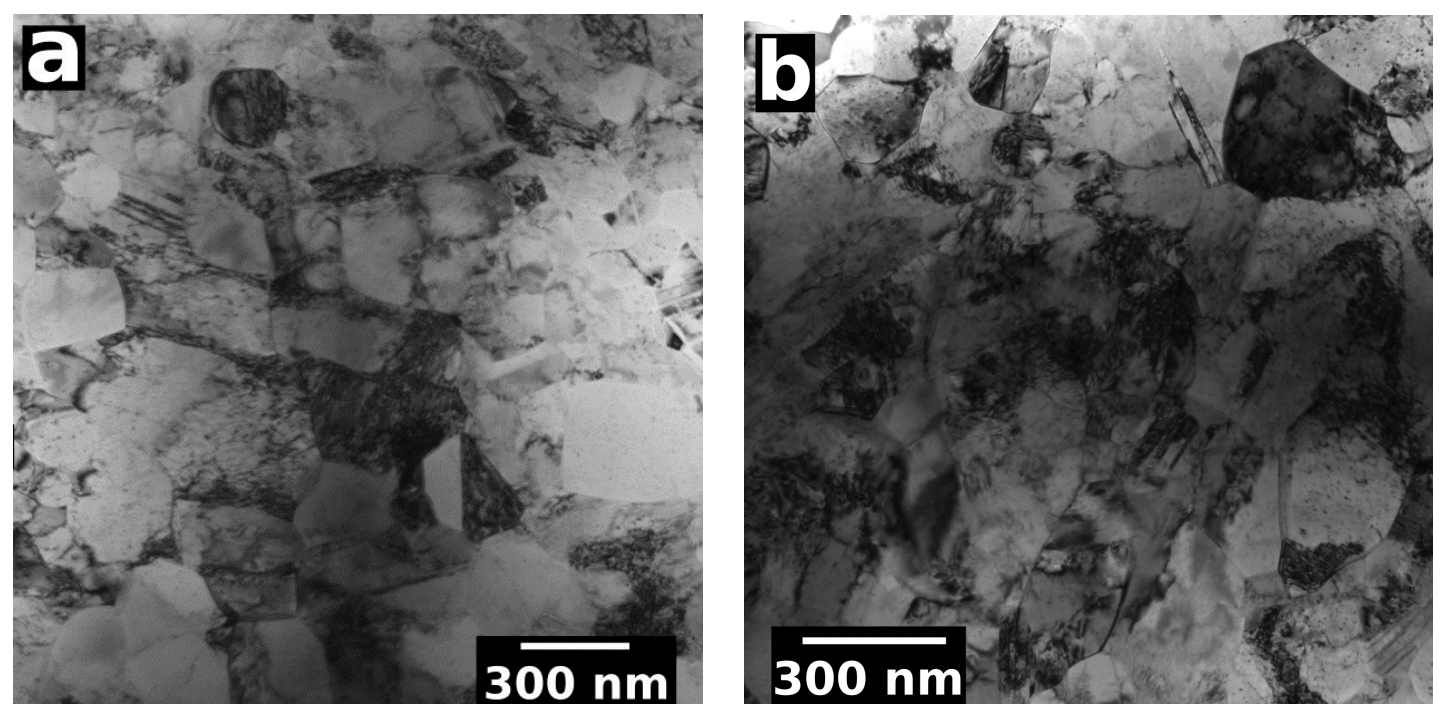

Figure 1. TEM images of 4N5 (a) and 4N (b) purity Ag samples processed by 8 passes of ECAP. The mean grain size is about $200 \mathrm{~nm}$ for both samples regardless of the impurity content.

Thermal Stability at Room Temperature. An earlier study [4] has shown that in 4N5 purity Ag samples self-annealing occurred during storage at RT. Figs. 2c plots the microhardness for 4N5 samples processed by 1-16 passes of ECAP as a function of the storage time at RT. After 1 pass in both 4N5 and 4N purity samples the hardness remained unchanged in the period of 4 months under review. At the same time, the decrease of the hardness with increasing time for $4 \mathrm{~N} 5$ purity specimens deformed by 4-16 passes clearly indicates a self-annealing. A former study [4] has revealed that both recovery and recrystallization occurred during storage of the ECAP-processed Ag samples. The larger the number of ECAP passes, the faster the hardness reduction for 4N5 purity samples (see Fig. 2c). Although, the dislocation density and therefore the driving force for recovery and recrystallization decreased between 8 and 16 passes, the fastest self-annealing was observed for the specimen processed by 16 passes. This can be explained by the highest twin boundary frequency among the studied samples as the strongly twinned volumes can act as nuclei for recrystallization thereby accelerating self-annealing [4]. The hardness versus storage time plot for 4N purity samples in Fig. 2d indicates that the two times larger impurity content resulted in a much higher stability that can be attributed to the pinning effect of impurities on dislocations and grainboundaries. 

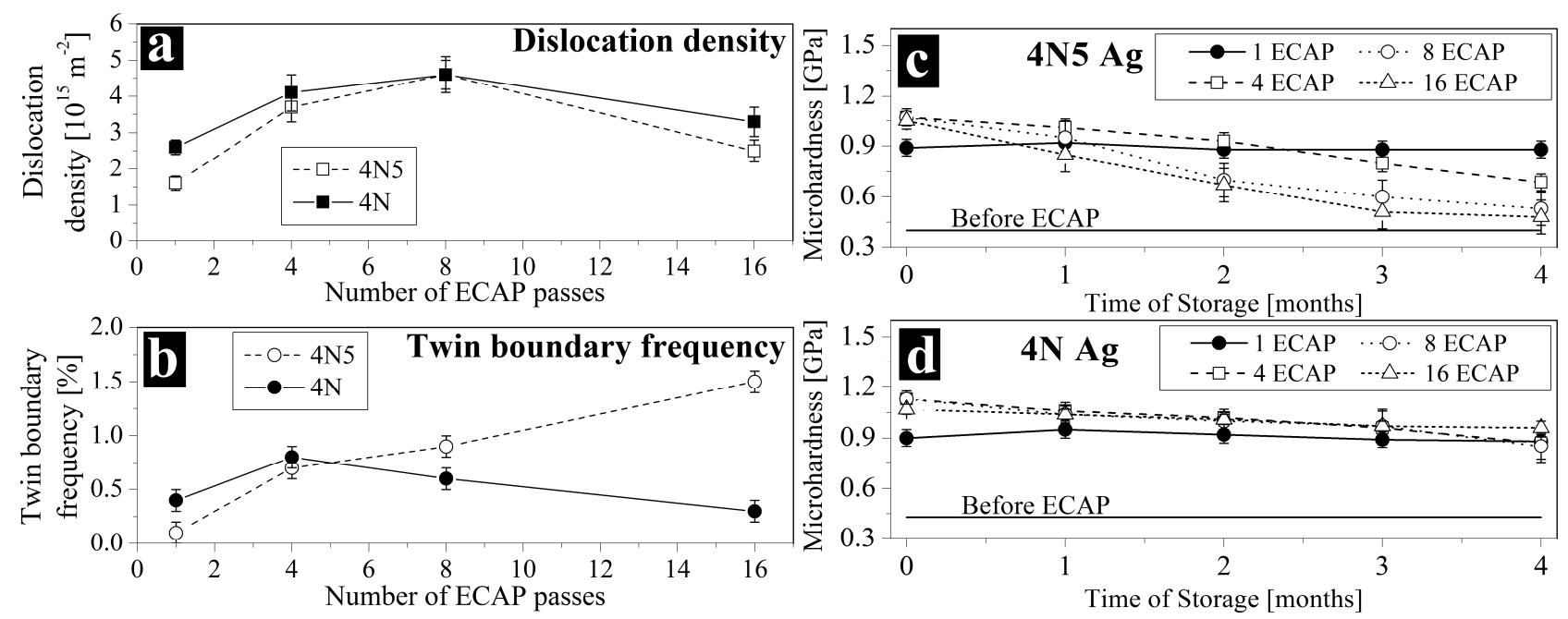

Figure 2. The variation of the dislocation density (a) and the twin boundary frequency (b) as a function of the number of ECAP passes for both 4N5 and 4N purity levels. Microhardness as a function of storage time for $4 \mathrm{~N} 5$ (c) and $4 \mathrm{~N}$ (d) purity samples processed by different numbers of ECAP passes. The microhardness values before ECAP-processing are also indicated in (c) and (d).

Thermal Stability at Elevated Temperatures. Fig. 3a shows the DSC thermograms measured at a heating rate of $10 \mathrm{~K} / \mathrm{min}$ for both $4 \mathrm{~N} 5$ and $4 \mathrm{~N}$ purity Ag samples processed by ECAP. An exothermic peak was observed for each sample that corresponds to the recovery and recrystallization of the ECAP-processed UFG microstructure. The maxima of the peaks are at the temperatures of $445 \mathrm{~K}, 408 \mathrm{~K}, 405 \mathrm{~K}$ and $412 \mathrm{~K}$ for $4 \mathrm{~N} 5$ purity samples and $488 \mathrm{~K}, 421 \mathrm{~K}, 421 \mathrm{~K}$ and $417 \mathrm{~K}$ for $4 \mathrm{~N}$ purity specimens processed by $1,4,8$ and 16 passes, respectively. It can be concluded that the temperature of recovery/recrystallization is much smaller for 4-16 passes than for 1 pass for both series due to the smaller grain size and the higher defect density that give a larger driving force for annealing processes. For each number of passes the temperature of peak maximum is higher while the released heat is lower for the 4N purity sample than for the 4N5 purity counterpart. This can be explained by the hindering effect of impurities on recovery and recrystallization. It is noted that in the case of 4N5 purity Ag, the high-temperature stability of the sample processed by 16 passes is not worse than for the specimens processed by 4 or 8 passes, while the self-annealing at RT was the fastest for the former sample as shown in the previous section. This can be explained by the large number of recrystallization nuclei formed at high temperatures that suppress the role of the twinned volumes developed during ECAP-processing.

It can be seen that Ag samples with higher impurity content exhibited lower released heat that can be explained by the pinning effect of solute atoms on lattice defects and therefore it is suspected that after DSC scans a considerable amount of lattice defects still remained in the materials. Indeed, the TEM image in Fig. 4b reveals a large density of dislocation loops appearing as dark spots in the picture taken after the DSC scan for the $4 \mathrm{~N}$ purity Ag sample processed by 4 passes. The volume density of the loops is about $8 \times 10^{22} \mathrm{~m}^{-3}$. A systematic analysis of a large number of images gave that 70 percent of the loops has $1 / 2<110>$ and 30 percent has $1 / 3<111>$ Burgers-vectors. The latter type of loops are vacancy agglomerates on $\{111\}$ lattice planes (Frank-loops). The formation of these vacancy clusters with large density during annealing is a consequence of the very low SFE in Ag since the interior of a Frank-loop is actually a stacking fault on $\{111\}$ lattice plane. Most probably, both types of dislocation loops were formed around the larger impurity atoms due to their attractive interactions. Therefore, it is a reasonable assumption that in $4 \mathrm{~N}$ purity samples the density of the remaining loops is much larger than in 4N5 purity counterparts that yields a smaller released heat during DSC experiments if the similar microstructures immediately after ECAP are taken into account. 

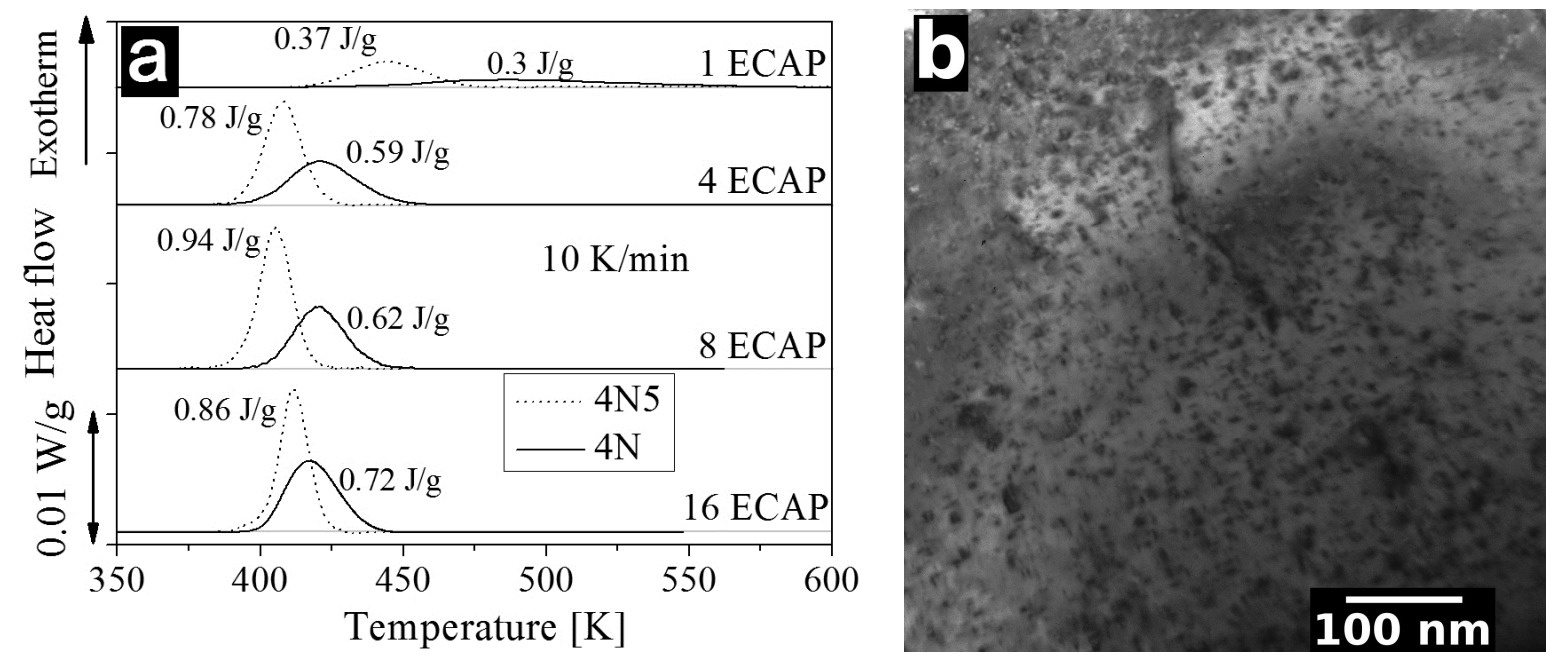

Figure 3. (a) DSC thermograms obtained at a heating rate of $10 \mathrm{~K} / \mathrm{min}$ for $4 \mathrm{~N} 5$ and $4 \mathrm{~N}$ purity $\mathrm{Ag}$ processed by different numbers of passes. The values at the exothermic peaks represent the released heat. (b) A bright field TEM image showing a large number of small dislocation-loops in the 4N purity sample processed by 4 passes and subsequently annealed up to the end of the exothermic peak on the DSC thermogram.

\section{Conclusions}

1. The results show that the minimum grain size in Ag achieved by ECAP at RT was $\sim 200 \mathrm{~nm}$ irrespective of the impurity atom concentration. The evolution of dislocation desity was also similar for both 4N5 and 4N purity levels. On the other hand, the evolution of twin boundary frequency was very different for the two impurity concentrations. As in UFG microstructures twins mainly form at grain-boundaries, the impurity segregation in the boundaries yielded lower twin boundary frequency in $4 \mathrm{~N}$ purity samples compared to $4 \mathrm{~N} 5$ purity counterparts for large number of ECAP passes.

2. The samples processed by 4-16 passes of ECAP showed a self-annealing during storage at RT. Despite the very small difference in impurity concentrations, $4 \mathrm{~N}$ purity samples showed much better stability during storage at RT than that for 4N5 purity counterparts. It seems that in addition to the pinning effect of impurities on lattice defects the smaller twin boundary frequency also stabilizes the UFG microstructure in $4 \mathrm{~N}$ purity specimens since strongly twinned volumes act as nuclei for recrystallization.

3. The exothermic DSC peaks for $4 \mathrm{~N}$ purity samples appeared at higher temperatures and the released heat values were smaller than in the case of $4 \mathrm{~N} 5$ purity specimens. The better hightemperature stability of $4 \mathrm{~N}$ purity samples can be explained by the hindering effect of impurities on recovery and recrystallization of the UFG microstructures. It was found that a large density of dislocation loops remained in the samples after the DSC peak. As these loops most probably formed around the large impurity atoms, the smaller released heat for $4 \mathrm{~N}$ purity samples can be attributed to the larger remaining energy stored in these loops.

\section{Acknowledgements}

This work was supported in part by the Hungarian Scientific Research Found, OTKA, Grant No. K81360 , in part by the National Science Foundation of the United States under Grant No. DMR0855009 and in part by the European Research Council under ERC Grant Agreement No. 267464SPDMETALS. The European Union and the European Social Found have provided financial support to this project under Grant Agreement No. TÁMOP 4.2.1./B-09/1/KMR-2010-0003. The authors thank Dr. Károly Havancsák, Gábor Varga and Andrea Jakab for taking the SEM images and preparation of the TEM samples. 


\section{References}

[1] R.Z. Valiev, T.G. Langdon, Principles of equal-channel angular pressing as a processing tool for grain refinement, Prog. Mater. Sci. 51 (2006) 881-981.

[2] A.P. Zhilyaev, T.G. Langdon, Using high-pressure torsion for metal processing: Fundamentals and applications, Prog. Mater. Sci. 53 (2008) 893-979.

[3] N. Q. Chinh, J. Gubicza, T. G. Langdon, Face-centered cubic metals and alloys processed by Equal-Channel Angular Pressing, J. Mater. Sci. 42 (2007) 1594-1605.

[4] J. Gubicza, N.Q. Chinh, J.L. Lábár, Z. Hegedűs, T.G. Langdon, Principles of self-annealing in silver processed by equal-channel angular pressing: The significance of a very low stacking fault energy, Mater. Sci. Eng. A 527 (2010) 752-760.

[5] D. Setman, E. Schafler, E. Korznikova, M.J. Zehetbauer, The presence and nature of vacancy type defects in nanometals detained by severe plastic deformation, Mater. Sci. Eng. A 493 (2008) 116-122.

[6] D. Setman, M.B. Kerber, E. Schafler, M.J. Zehetbauer, Activation Enthalpies of DeformationInduced Lattice Defects in Severe Plastic Deformation Nanometals Measured by Differential Scanning Calorimetry, Metall. Mater. Trans. A 41 (2010) 810-815.

[7] M. Furukawa, Y. Iwahashi, Z. Horita, M. Nemoto, T.G. Langdon, The shearing characteristics associated with equal-channel angular pressing, Mater. Sci. Eng. A 257 (1998) 328-332.

[8] Y. Iwahashi, J. Wang, Z. Horita, M. Nemoto, T.G. Langdon, Principle of equal-channel angular pressing for the processing of ultra-fine grained materials, Scripta Mater. 35 (1996) 143-146.

[9] G. Ribárik, J. Gubicza, T. Ungár, Correlation between strength and microstructure of ball milled Al-Mg alloys determined by X-ray diffraction, Mater. Sci. Eng. A 387-389 (2004) 343347.

[10] L. Balogh, G. Ribárik, T. Ungár, Stacking faults and twin boundaries in fcc crystals determined by x-ray diffraction profile analysis, J. Appl. Phys. 100 (2006) 023512.

[11] E. Schafler, G. Steiner, E. Korznikova, M. Kerber, M.J. Zehetbauer, Lattice defect investigation of ECAP-Cu by means of X-ray line profile analysis, calorimetry and electrical resistometry, Mater. Sci. Eng. A 410-411 (2005) 169-173.

[12] J.P. Hirth, J. Lothe, Theory of Dislocations, Wiley, New York, 1982.

[13] L.E. Murr, Interfacial Phenomena in Metals and Alloys, Addison Wesley, Reading, MA, 1975.

[14] Y.T. Zhu, X.L. Wu, X.Z. Liao, J. Narayan, S.N. Mathaudhu, L.J. Kecskés, Twinning partial multiplication at grain boundary in nanocrystalline fcc metals, Appl. Phys. Lett. 95 (2009) 031909.

[15] P.V. Liddicoat, X.-Z. Liao, Y. Zhao, Y. Zhu, M.Y. Murashkin, E.J. Lavernia, R.Z. Valiev, S.P. Ringer, Nanostructural hierarchy increases the strength of aluminium alloys, Nat. Commun. 1 (2010) 63. 